\title{
The fourth power mean of the generalized two-term exponential sums and its upper and lower bound estimates
}

Xiaoxue Li $i^{*}$ and Zhefeng Xu

\section{"Correspondence:} Ixx20072012@163.com Department of Mathematics, Northwest University, Xi'an, Shaanxi, P.R. China

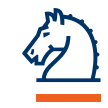

\begin{abstract}
In this paper, we use the analytic method and the properties of Gauss sums to study the computational problem of one kind fourth power mean of the generalized two-term exponential sums, and give an exact computational formula for it.

MSC: Primary 11L40; 11F20

Keywords: generalized two-term exponential sums; fourth power mean; computational formula
\end{abstract}

\section{Introduction}

Let $q \geq 3$ be a positive integer. For any integers $m$ and $n$, the generalized two-term exponential sum $C(m, n, k, \chi ; q)$ is defined by

$$
C(m, n, k, \chi ; q)=\sum_{a=1}^{q} \chi(a) e\left(\frac{m a^{k}+n a}{q}\right),
$$

where $\chi$ denotes any Dirichlet character $\bmod q$, and $e(y)=e^{2 \pi i y}$.

Regarding the upper bound estimate of $C(m, n, k, \chi ; q)$, many authors have studied it and obtained a series of important results; related contents can be found in [1-5] and [6]. For example, from Weil's classical work [7] one can deduce the estimate

$$
|C(m, 0,2, \chi ; p)| \leq 2 \cdot p^{\frac{1}{2}}
$$

for $(m, p)=1$.

Recently, Wang [8] studied the computational problem of the fourth power mean of $C(m, n, k, \chi ; p)$, and proved the following conclusion:

Let $p$ be an odd prime with $p \neq 3 a+1$. Then, for any integer $m$ with $(m, p)=1$, we have the identity

$$
\begin{aligned}
& \sum_{n=1}^{p}|C(m, n, 3, \chi ; p)|^{4} \\
& \quad= \begin{cases}p\left(2 p^{2}-3 p-3\right) & \text { if } \chi \text { is the principal character mod } p ; \\
p^{2}(3 p-7) & \text { if } \chi \text { is the Legendre symbol } \bmod p ; \\
p^{2}(2 p-6) & \text { otherwise. }\end{cases}
\end{aligned}
$$

(2013 Li and Xu; licensee Springer. This is an Open Access article distributed under the terms of the Creative Commons Attribution License (http://creativecommons.org/licenses/by/2.0), which permits unrestricted use, distribution, and reproduction in any medium, provided the original work is properly cited. 
Wang [9] studied the hybrid power mean of the generalized Kloosterman sums $\sum_{a=1}^{p-1} \lambda(a) \times$ $e\left(\frac{m a+\bar{a}}{p}\right)$ and $\sum_{a=1}^{p-1} \chi(a+\bar{a})$, where $\lambda$ denotes a Dirichlet character mod $p$, and gave an interesting asymptotic formula for it. That is, she proved the following result:

Let $p$ be an odd prime. Then, for any non-principal even character $\chi \bmod p$ and any character $\lambda \bmod p$ with $\lambda \neq\left(\frac{*}{p}\right)$, we have the asymptotic formula

$$
\sum_{m=1}^{p-1}\left|\sum_{a=1}^{p-1} \lambda(a) e\left(\frac{m a+\bar{a}}{p}\right)\right|^{2} \cdot\left|\sum_{b=1}^{p-1} \chi(m b+\bar{b})\right|^{2}=2 p^{3}+O\left(p^{2}\right) .
$$

In this paper, as a note of [8] and [9], we found that there exists a close relationship between the fourth power mean of $C(m, n, 2, \chi ; p)$ and $\left|\sum_{b=1}^{p-1} \chi(n b+\bar{b})\right|$. The main purpose of this paper is to show this point. That is, we shall prove the following theorem.

Theorem Let $p$ be an odd prime. Then, for any character $\chi_{1} \bmod p$, we have the identity

$$
\begin{aligned}
\sum_{m=1}^{p} \mid & \left|\sum_{a=1}^{p-1} \chi_{1}(a) e\left(\frac{m a^{2}+a}{p}\right)\right|^{4} \\
& = \begin{cases}p^{3}-3 p^{2}+2\left(\frac{-1}{p}\right) p^{2}-p-8\left(\frac{-1}{p}\right) p & \text { if } \chi_{1}=\chi_{0} ; \\
2 p^{3}-3 p^{2} & \text { if } \chi_{1}(-1)=-1 ; \\
2 p^{3}-4\left(\frac{-1}{p}\right) \cdot p^{2}-3 p^{2}-p \cdot\left|\sum_{a=1}^{p-1} \chi_{1}(a+\bar{a})\right|^{2} & \text { if } \chi_{1} \neq \chi_{0} \text { and } \chi_{1}(-1)=1,\end{cases}
\end{aligned}
$$

where $\chi_{0}$ denotes the principal character $\bmod p, a \cdot \bar{a} \equiv 1 \bmod p$, and $\left(\frac{*}{p}\right)$ is the Legendre symbol.

From this theorem we may immediately deduce the following corollary.

Corollary Let $p$ be an odd prime. Then, for any non-principal character $\chi_{1} \bmod p$, we have the inequalities

$$
2 p^{3}-11 p^{2} \leq \sum_{m=1}^{p}\left|\sum_{a=1}^{p-1} \chi_{1}(a) e\left(\frac{m a^{2}+a}{p}\right)\right|^{4} \leq 2 p^{3}+p^{2} .
$$

\section{Several lemmas}

In this section, we shall give several lemmas, which are necessary in the proof of our theorem. Hereinafter, we shall use many properties of character sums and Gauss sums, all of these can be found in reference [10], so they will not be repeated here. First we have the following.

Lemma 1 Let $p$ be an odd prime. Then, for any integers $m$ and $n$ with $(m n, p)=1$, we have the identity

$$
\sum_{b=0}^{p-1} e\left(\frac{m b^{2}+n b}{p}\right)=\left(\frac{m}{p}\right) e\left(\frac{-\overline{4 m} n^{2}}{p}\right) \sum_{a=0}^{p-1} e\left(\frac{a^{2}}{p}\right),
$$

where $\left(\frac{x}{p}\right)$ denotes the Legendre symbol, and $\bar{m} m \equiv 1 \bmod p$. 
Proof See Lemma 1 in [8].

Lemma 2 Let $p$ be an odd prime, $\chi_{1}$ be any fixed character mod $p$. Then, for any non-real character $\chi \bmod p$, we have the identity

$$
\begin{gathered}
\left.\left.\left|\sum_{m=1}^{p-1} \chi(m)\right| \sum_{a=1}^{p-1} \chi_{1}(a) e\left(\frac{m a^{2}+a}{p}\right)\right|^{2}\right|^{2} \\
=p^{2} \cdot\left|\sum_{a=1}^{p-1} \chi_{1}(a+1) \bar{\chi}(1+2 \bar{a})\right|^{2} .
\end{gathered}
$$

Proof From the properties of Gauss sums, we have

$$
\begin{aligned}
& \sum_{m=1}^{p-1} \chi(m)\left|\sum_{a=1}^{p-1} \chi_{1}(a) e\left(\frac{m a^{2}+a}{p}\right)\right|^{2} \\
& =\sum_{a=1}^{p-1} \sum_{b=1}^{p-1} \chi_{1}(a \bar{b}) \sum_{m=1}^{p-1} \chi(m) e\left(\frac{m\left(a^{2}-b^{2}\right)+(a-b)}{p}\right) \\
& =\sum_{a=1}^{p-1} \sum_{b=1}^{p-1} \chi_{1}(a) \sum_{m=1}^{p-1} \chi(m) e\left(\frac{m b^{2}\left(a^{2}-1\right)+b(a-1)}{p}\right) \\
& =\tau(\chi) \sum_{a=1}^{p-1} \chi_{1}(a) \sum_{b=1}^{p-1} \bar{\chi}\left(b^{2}\left(a^{2}-1\right)\right) e\left(\frac{b(a-1)}{p}\right) \\
& =\tau(\chi) \tau\left(\bar{\chi}^{2}\right) \sum_{a=1}^{p-1} \chi_{1}(a) \bar{\chi}\left(a^{2}-1\right) \chi^{2}(a-1) \\
& =\tau(\chi) \tau\left(\bar{\chi}^{2}\right) \sum_{a=2}^{p-1} \chi_{1}(a) \bar{\chi}(a+1) \chi(a-1) \\
& =\tau(\chi) \tau\left(\bar{\chi}^{2}\right) \sum_{a=1}^{p-1} \chi_{1}(a+1) \bar{\chi}(1+2 \bar{a}) .
\end{aligned}
$$

Note that $\chi_{1}(p-1+1)=0, \chi$ is a non-real character $\bmod p$, so $\bar{\chi}^{2}$ is also a non-principal character $\bmod p$. Therefore, $|\tau(\chi)|=\left|\tau\left(\bar{\chi}^{2}\right)\right|=\sqrt{p}$, so from (1) we may immediately deduce Lemma 2.

Lemma 3 Let $p$ be an odd prime, $\chi$ be any non-principal character $\bmod p$ with $\chi(-1)=1$. Then we have

$$
\left|\sum_{a=1}^{p-1} \chi(a+\bar{a})\right|^{2}=\left(\frac{-1}{p}\right) \cdot\left(\sum_{a=1}^{p-1} \chi(a)\left(\frac{a^{2}-1}{p}\right)\right)^{2}
$$

and

$$
\left|\sum_{a=1}^{p-1} \chi(a+\bar{a})\right| \leq 2 \cdot \sqrt{p}
$$


Proof From the properties of quadratic residue $\bmod p$, we have

$$
\begin{aligned}
\sum_{a=1}^{p-1} \chi(a+\bar{a}) & =\sum_{b=1}^{p-1} \chi(b) \sum_{\substack{a=1 \\
a+\bar{a} \equiv b \bmod p}}^{p-1} 1=\sum_{b=1}^{p-1} \chi(b) \sum_{\substack{a=0 \\
a^{2}-b a+1 \equiv 0 \bmod p}}^{p-1} 1 \\
& =\sum_{b=1}^{p-1} \chi(b) \quad \sum_{\substack{(2 a-b)^{2} \equiv b^{2}-4 \bmod p \\
p-1}}^{p-1} \chi(2) \sum_{b=1}^{p-1} \chi(b) \sum_{a=0}^{p-1} 1 \\
& =\chi(2) \cdot \sum_{b=1}^{p-1} \chi(b)\left(1+\left(\frac{b^{2}-1}{p}\right)\right)=\chi(2) \cdot \sum_{b=1}^{p-1} \chi(b)\left(\frac{b^{2}-1}{p}\right) .
\end{aligned}
$$

Note that

$$
\sum_{b=1}^{p-1} \bar{\chi}(b)\left(\frac{b^{2}-1}{p}\right)=\sum_{b=1}^{p-1} \chi(b)\left(\frac{\bar{b}^{2}-1}{p}\right)=\left(\frac{-1}{p}\right) \sum_{b=1}^{p-1} \chi(b)\left(\frac{b^{2}-1}{p}\right)
$$

so from (2) we may immediately deduce the identity

$$
\left|\sum_{a=1}^{p-1} \chi(a+\bar{a})\right|^{2}=\left(\frac{-1}{p}\right)\left(\sum_{a=1}^{p-1} \chi(a)\left(\frac{a^{2}-1}{p}\right)\right)^{2} .
$$

The estimate

$$
\left|\sum_{a=1}^{p-1} \chi(a+\bar{a})\right| \leq 2 \cdot \sqrt{p}
$$

follows from Lemma 1 of [9]. This proves Lemma 3.

\section{Proof of Theorem}

In this section, we shall give two different proofs of our theorem. First, if $\chi_{1}$ is a nonprincipal character mod $p$, then from Lemma 1 we have

$$
\begin{aligned}
& \left|\sum_{a=1}^{p-1} \chi_{1}(a) e\left(\frac{m a^{2}+a}{p}\right)\right|^{2} \\
& \quad=\sum_{a=1}^{p-1} \sum_{b=1}^{p-1} \chi_{1}(a \bar{b}) e\left(\frac{m\left(a^{2}-b^{2}\right)+a-b}{p}\right) \\
& \quad=\sum_{a=1}^{p-1} \sum_{b=1}^{p-1} \chi_{1}(a) e\left(\frac{m b^{2}\left(a^{2}-1\right)+b(a-1)}{p}\right) \\
& \quad=p-1+\chi_{1}(-1) \sum_{b=1}^{p-1} e\left(\frac{-2 b}{p}\right)+\sum_{a=2}^{p-2} \chi_{1}(a) \sum_{b=1}^{p-1} e\left(\frac{m b^{2}\left(a^{2}-1\right)+b(a-1)}{p}\right) \\
& \quad=p-1-\chi_{1}(-1)+\sum_{a=2}^{p-2} \chi_{1}(a) \sum_{b=0}^{p-1} e\left(\frac{m b^{2}\left(a^{2}-1\right)+b(a-1)}{p}\right)-\sum_{a=2}^{p-2} \chi_{1}(a)
\end{aligned}
$$


Li and Xu Journal of Inequalities and Applications 2013, 2013:504

Page 5 of 8

http://www.journalofinequalitiesandapplications.com/content/2013/1/504

$$
\begin{aligned}
& =p+\sum_{a=2}^{p-2} \chi_{1}(a) \sum_{b=0}^{p-1} e\left(\frac{m(a+1) \overline{a-1} \cdot b^{2}+b}{p}\right)-\sum_{a=1}^{p-1} \chi_{1}(a) \\
& =p+G(p) \cdot \sum_{a=2}^{p-2} \chi_{1}(a)\left(\frac{m(a+1) \overline{a-1}}{p}\right) e\left(\frac{\overline{4 m} \cdot \overline{a+1}(a-1)}{p}\right) \\
& =p+G(p) \cdot \sum_{a=2}^{p-2} \chi_{1}(a)\left(\frac{m\left(a^{2}-1\right)}{p}\right) e\left(\frac{\overline{4 m} \cdot \overline{a+1}(a-1)}{p}\right),
\end{aligned}
$$

where $G(p)=\sum_{a=0}^{p-1} e\left(\frac{a^{2}}{p}\right)$ and $G^{2}(p)=\left(\frac{-1}{p}\right) \cdot p$ (see Theorem 7.5.4 of [5]).

From (3) and the definition of Gauss sums, we may immediately deduce

$$
\begin{aligned}
\sum_{m=1}^{p-1} \mid & \left.\sum_{a=1}^{p-1} \chi_{1}(a) e\left(\frac{m a^{2}+a}{p}\right)\right|^{4} \\
= & p^{2}(p-1)+2 p G(p) \sum_{m=1}^{p-1} \sum_{a=2}^{p-2} \chi_{1}(a)\left(\frac{m\left(a^{2}-1\right)}{p}\right) e\left(\frac{\overline{4 m} \cdot \overline{a+1}(a-1)}{p}\right) \\
& +G^{2}(p) \sum_{a=2}^{p-2} \sum_{b=2}^{p-2} \chi_{1}(a b)\left(\frac{\left(a^{2}-1\right)\left(b^{2}-1\right)}{p}\right) \\
& \times \sum_{m=1}^{p-1} e\left(\frac{4 m \cdot(\overline{a+1}(a-1)+\overline{b+1}(b-1))}{p}\right) \\
= & p^{2}(p-1)+2 p G^{2}(p) \sum_{a=2}^{p-2} \chi_{1}(a)\left(\frac{\left(a^{2}-1\right)(a-1) \overline{a+1}}{p}\right) \\
& +p G^{2}(p) \sum_{a=2}^{p-2} \chi_{1}(1)\left(\frac{\left(a^{2}-1\right)\left(\bar{a}^{2}-1\right)}{p}\right)-G^{2}(p)\left(\sum_{a=2}^{p-2} \chi_{1}(a)\left(\frac{a^{2}-1}{p}\right)\right)^{2} \\
= & p^{2}(p-1)+2 p G^{2}(p) \sum_{a=2}^{p-2} \chi_{1}(a)+p G^{2}(p)\left(\frac{-1}{p}\right)(p-3) \\
& \left.-\sum_{a=2}^{p-2} \chi_{1}(a)\left(\frac{a^{2}-1}{p}\right)\right)^{2} \cdot(p)
\end{aligned}
$$

If $\chi_{1}$ is a non-principal character $\bmod p$ with $\chi_{1}(-1)=-1$, then note that

$$
\begin{aligned}
& \sum_{a=2}^{p-2} \chi_{1}(a)\left(\frac{a^{2}-1}{p}\right)=\sum_{a=1}^{p-1} \chi_{1}(a)\left(\frac{a^{2}-1}{p}\right)=0, \\
& G^{2}(p)=\left(\sum_{a=0}^{p-1} e\left(\frac{a^{2}}{p}\right)\right)^{2}=\left(\frac{-1}{p}\right) \cdot p
\end{aligned}
$$

and

$$
\left|\sum_{a=1}^{p-1} \chi_{1}(a) e\left(\frac{0 \cdot a^{2}+a}{p}\right)\right|^{4}=\left|\sum_{a=1}^{p-1} \chi_{1}(a) e\left(\frac{a}{p}\right)\right|^{4}=p^{2} .
$$


From (4) we have

$$
\sum_{m=0}^{p-1}\left|\sum_{a=1}^{p-1} \chi_{1}(a) e\left(\frac{m a^{2}+a}{p}\right)\right|^{4}=2 p^{3}-3 p^{2}
$$

If $\chi_{1}$ is a non-principal character $\bmod p$ with $\chi_{1}(-1)=1$, then from (4) and Lemma 3 we have

$$
\begin{aligned}
& \sum_{m=0}^{p-1}\left|\sum_{a=1}^{p-1} \chi_{1}(a) e\left(\frac{m a^{2}+a}{p}\right)\right|^{4} \\
& \quad=2 p^{3}-4\left(\frac{-1}{p}\right) \cdot p^{2}-3 p^{2}-\left(\frac{-1}{p}\right) \cdot p \cdot\left(\sum_{a=1}^{p-1} \chi_{1}(a)\left(\frac{a^{2}-1}{p}\right)\right)^{2} \\
& \quad=2 p^{3}-4\left(\frac{-1}{p}\right) \cdot p^{2}-3 p^{2}-p \cdot\left|\sum_{a=1}^{p-1} \chi_{1}(a+\bar{a})\right|^{2}
\end{aligned}
$$

If $\chi_{1}=\chi_{0}$ is the principal character $\bmod p$, then from the method of proving (3) and (4) we have

$$
\sum_{m=0}^{p-1}\left|\sum_{a=1}^{p-1} e\left(\frac{m a^{2}+a}{p}\right)\right|^{4}=p^{3}-3 p^{2}+2\left(\frac{-1}{p}\right) p^{2}-p-8\left(\frac{-1}{p}\right) p .
$$

Combining (5), (6) and (7), we may immediately deduce our theorem.

The second proof of Theorem. First, from the orthogonality of characters $\bmod p$, we have

$$
\begin{gathered}
\left.\left.\sum_{\chi \bmod p}\left|\sum_{m=1}^{p-1} \chi(m)\right| \sum_{a=1}^{p-1} \chi_{1}(a) e\left(\frac{m a^{2}+a}{p}\right)\right|^{2}\right|^{2} \\
=(p-1) \cdot \sum_{m=1}^{p-1}\left|\sum_{a=1}^{p-1} \chi_{1}(a) e\left(\frac{m a^{2}+a}{p}\right)\right|^{4}
\end{gathered}
$$

On the other hand, from Lemma 2 we have

$$
\begin{aligned}
& \left.\left.\sum_{\chi \bmod p}\left|\sum_{m=1}^{p-1} \chi(m)\right| \sum_{a=1}^{p-1} \chi_{1}(a) e\left(\frac{m a^{2}+a}{p}\right)\right|^{2}\right|^{2} \\
& =p^{2} \cdot \sum_{\chi \bmod p}\left|\sum_{a=1}^{p-1} \chi_{1}(a+1) \bar{\chi}(1+2 \bar{a})\right|^{2}+\left(\sum_{m=1}^{p-1}\left|\sum_{a=1}^{p-1} \chi_{1}(a) e\left(\frac{m a^{2}+a}{p}\right)\right|^{2}\right)^{2} \\
& +\left.\left.\left|\sum_{m=1}^{p-1}\left(\frac{m}{p}\right)\right| \sum_{a=1}^{p-1} \chi_{1}(a) e\left(\frac{m a^{2}+a}{p}\right)\right|^{2}\right|^{2}-p^{2} \cdot\left|\sum_{a=1}^{p-2} \chi_{1}(a+1) \chi_{0}(1+2 \bar{a})\right|^{2} \\
& -p^{2} \cdot\left|\sum_{a=1}^{p-2} \chi_{1}(a+1)\left(\frac{1+2 \bar{a}}{p}\right)\right|^{2} \\
& \equiv p^{2} A+B+C-p^{2} D-p^{2} E .
\end{aligned}
$$


Applying the orthogonality of characters $\bmod p$, we can easily deduce that

$$
\begin{aligned}
A & =\sum_{\chi \bmod p}\left|\sum_{a=1}^{p-1} \chi_{1}(a+1) \bar{\chi}(1+2 \bar{a})\right|^{2}=(p-1)(p-3), \\
B & =\left(\sum_{m=1}^{p-1}\left|\sum_{a=1}^{p-1} \chi_{1}(a) e\left(\frac{m a^{2}+a}{p}\right)\right|^{2}\right)^{2} \\
& = \begin{cases}\left(p^{2}-2 p-1\right)^{2} & \text { if } \chi_{1}=\chi_{0} \\
p^{2}\left(p-2-\chi_{1}(-1)\right)^{2} & \text { if } \chi_{1} \neq \chi_{0} .\end{cases}
\end{aligned}
$$

From the definition and properties of Gauss sums, we have

$$
\begin{aligned}
C & =\left.\left.\left|\sum_{m=1}^{p-1}\left(\frac{m}{p}\right)\right| \sum_{a=1}^{p-1} \chi_{1}(a) e\left(\frac{m a^{2}+a}{p}\right)\right|^{2}\right|^{2}=p \cdot\left|\sum_{a=1}^{p-1} \chi_{1}(a)\left(\frac{a^{2}-1}{p}\right)\right|^{2}, \\
D & =\left|\sum_{a=1}^{p-2} \chi_{1}(a+1) \chi_{0}(1+2 \bar{a})\right|^{2}=p-3, \\
E & =\left|\sum_{a=1}^{p-2} \chi_{1}(a+1)\left(\frac{1+2 \bar{a}}{p}\right)\right|^{2}=\left|\sum_{a=1}^{p-1} \chi_{1}(a)\left(\frac{a^{2}-1}{p}\right)\right|^{2} .
\end{aligned}
$$

Note that if $\chi_{1}(-1)=-1$, then

$$
\sum_{a=1}^{p-1} \chi_{1}(a)\left(\frac{a^{2}-1}{p}\right)=0 .
$$

Combining (7)-(14) and Lemma 3, we may immediately deduce the identity

$$
\begin{aligned}
& \sum_{m=1}^{p-1}\left|\sum_{a=1}^{p-1} \chi_{1}(a) e\left(\frac{m a^{2}+a}{p}\right)\right|^{4} \\
& \quad= \begin{cases}p^{3}-3 p^{2}+2\left(\frac{-1}{p}\right) p^{2}-p-8\left(\frac{-1}{p}\right) p-1 & \text { if } \chi_{1}=\chi_{0} ; \\
2 p^{3}-4 p^{2} & \text { if } \chi_{1}(-1)=-1 ; \\
2 p^{3}-4\left(\frac{-1}{p}\right) \cdot p^{2}-4 p^{2}-p \cdot\left|\sum_{a=1}^{p-1} \chi_{1}(a+\bar{a})\right|^{2} & \text { if } \chi_{1} \neq \chi_{0} \text { and } \chi_{1}(-1)=1 .\end{cases}
\end{aligned}
$$

This completes another proof of our theorem.

The corollary follows from Theorem and Lemma 3.

\section{Competing interests}

The authors declare that they have no competing interests.

\section{Authors' contributions}

LX carried out the part of Introduction, XZ carried out the proof of some lemmas, LX with XZ carried out the theorem's proof. All authors read and approved the final manuscript.

\section{Acknowledgements}

The authors would like to thank the referee for his/her very helpful and detailed comments, which have significantly improved the presentation of this paper. This work is supported by the N.S.F. (11071194) of P.R. China. 


\section{References}

1. Cochrane, T, Zheng, Z: Upper bounds on a two-term exponential sums. Sci. China Ser. A 44, 1003-1015 (2001)

2. Cochrane, T, Zheng, ZY: Pure and mixed exponential sums. Acta Arith. 91, 249-278 (1999)

3. Cochrane, T, Pinner, C: A further refinement of Mordell's bound on exponential sums. Acta Arith. 116, 35-41 (2005)

4. Cochrane, T, Pinner, C: Using Stepanov's method for exponential sums involving rational functions. J. Number Theory $116,270-292(2006)$

5. Hua, LK: Introduction to Number Theory. Science Press, Beijing (1979)

6. Zhang, W: Moments of generalized quadratic Gauss sums weighted by L-functions. J. Number Theory 92, 304-314 (2002)

7. Weil, A: On some exponential sums. Proc. Natl. Acad. Sci. USA 34, 204-207 (1948)

8. Wang, T: On the fourth power mean of generalized two-term exponential sums. Bull. Korean Math. Soc. 50, 233-240 (2013)

9. Wang, J: Hybrid mean value of the generalized Kloosterman sums and Dirichlet character of polynomials. Bull. Korean Math. Soc. 50, 451-458 (2013)

10. Apostol, TM: Introduction to Analytic Number Theory. Springer, New York (1976)

10.1186/1029-242X-2013-504

Cite this article as: $\mathrm{Li}$ and $\mathrm{Xu}$ : The fourth power mean of the generalized two-term exponential sums and its upper and lower bound estimates. Journal of Inequalities and Applications 2013, 2013:504

\section{Submit your manuscript to a SpringerOpen ${ }^{\ominus}$ journal and benefit from:}

- Convenient online submission

- Rigorous peer review

- Immediate publication on acceptance

- Open access: articles freely available online

- High visibility within the field

- Retaining the copyright to your article 